\title{
Bounds for the singular values of Schwarz matrix
}

\author{
Cecília Costa
}




\title{
BOUNDS FOR THE SINGULAR VALUES OF THE SCHWARZ MATRIX
}

\author{
CECÍLIA COSTA
}

[Received: September 7, 2003]

\begin{abstract}
Aвstract. Upper bounds for the squares of singular values of the Schwarz matrix are obtained by using matricial (and matrix) norms and inequalities concerning the spectral radii of matrices.
\end{abstract}

Mathematics Subject Classification: 15A18, 15A42

Keywords: Singular values, matricial norms, matrix norms, Schwarz matrix

\section{InTRODUCTION}

Recently, singular values and singular value decomposition have attracted much attention because of their applications in various areas, namely, in control theory and systems theory [5], in biomedical engineering [3], and in signal and image processing. The application of singular values is illustrated by examples in controllability and observability problems [5], in obtaining the fetal ECG from that of the mother [3], and in processing, storage and retrieval of information [2].

Here, we are interested in establishing bounds for the singular values of the Schwarz matrix, $S$.

A special choice of the elements of $S$ (involving Hurwitz determinants) guarantees that the characteristic polynomial of $S$ is a given polynomial $a(x)$ [1, p. 211]. In this case, it can be shown that $S$ is similar to the usual companion matrix associated with $a(x)$. Thus, the Schwarz matrix is a real canonical form to which any non-degenerate matrix is similar [1, p. 212]. However, such form of $S$ is not relevant for the purpose of this paper, so hereafter we consider the Schwarz matrix, $S$ (of order $m$ ) given as

Work done at the Grupo de Teoria do Controlo, Instituto de Sistemas e Robótica, Pólo de Coimbra, 3000 Coimbra, Portugal. 
follows:

$$
S=\left[\begin{array}{ccccc}
0 & 1 & 0 & \cdots & 0 \\
-s_{m} & 0 & \ddots & \ddots & \vdots \\
0 & -s_{m-1} & \ddots & \ddots & 0 \\
\vdots & \ddots & \ddots & 0 & 1 \\
0 & \cdots & 0 & -s_{2} & -s_{1}
\end{array}\right]
$$

with $s_{i} \in \mathbb{R}(i=1, \ldots, m)$. The Schwarz matrix is of interest in its own right [1, p. 212]. It is known that this matrix satisfies the Lyapunov equation (see [1, p. 208] and $[9, \mathrm{p} .450]$ ). It is also proved that (depending on the normalizing factor) the continuant matrix associated with a certain polynomial recurrence involving Hurwitz polynomials, has the Schwarz form [8].

Such a matrix, nowadays referred to as the Schwarz matrix, was first presented in [12] and is used extensively in control theory [8]. It also appears in circuit theory under the name "Bückner matrix" [8].

The tools used in this paper are the matricial and matrix norms and inequalities concerning the spectral radius of a matrix and the spectral radius of a convenient matricial norm $[6,7,11]$.

\section{Results}

Singular values, $v_{j}(j=1, \ldots, m)$, of $S$, are the positive square roots of the eigenvalues of $S^{T} S$. Thus it is useful to make known the structure of such a matrix, which we present in the following Lemma.

Lemma 1. The matrix $S^{T} S$ associated with Schwarz matrix $S$, of order $m \geq 5$, is of the form

$$
S^{T} S=\left[\begin{array}{cc}
P & Q \\
Q^{T} & R
\end{array}\right]
$$

where $P$ is the following symmetric matrix:

$$
P=\left[\begin{array}{cccccc}
s_{m}^{2} & 0 & -s_{m} & 0 & \cdots & 0 \\
0 & 1+s_{m-1}^{2} & 0 & -s_{m-1} & \ddots & \vdots \\
-s_{m} & 0 & \ddots & \ddots & \ddots & 0 \\
0 & -s_{m-1} & \ddots & \ddots & 0 & -s_{5} \\
\vdots & \ddots & \ddots & 0 & 1+s_{4}^{2} & 0 \\
0 & \cdots & 0 & -s_{5} & 0 & 1+s_{3}^{2}
\end{array}\right] \in Q_{m-2}(\mathbb{R})
$$

$Q=\left[\begin{array}{c}O \\ Q_{F}\end{array}\right] \in Q_{(m-2) \times 2}(\mathbb{R})$, with $Q_{F}=\left[\begin{array}{cc}-s_{4} & 0 \\ 0 & -s_{3}\end{array}\right], O$ the null matrix of order $(m-4) \times 2$, and $R=\left[\begin{array}{cc}1+s_{2}^{2} & s_{1} s_{2} \\ s_{1} s_{2} & 1+s_{1}^{2}\end{array}\right] \in Q_{2}(\mathbb{R})$. 
Proof. The proof goes by induction with respect to the order of the matrix $S$.

Remark 1 . If the order of $S$ is $l=2,3,4$, elementary calculations show that $S_{l}^{T} S_{l}$ is given by the formulae

$$
S_{2}^{T} S_{2}=\left[\begin{array}{cc}
s_{2}^{2} & s_{1} s_{2} \\
s_{1} s_{2} & 1+s_{1}^{2}
\end{array}\right], \quad S_{3}^{T} S_{3}=\left[\begin{array}{ccc}
s_{3}^{2} & 0 & -s_{3} \\
0 & 1+s_{2}^{2} & s_{1} s_{2} \\
-s_{3} & s_{1} s_{2} & 1+s_{1}^{2}
\end{array}\right],
$$

and

$$
S_{4}^{T} S_{4}=\left[\begin{array}{cccc}
s_{4}^{2} & 0 & -s_{4} & 0 \\
0 & 1+s_{3}^{2} & 0 & -s_{3} \\
-s_{4} & 0 & 1+s_{2}^{2} & s_{1} s_{2} \\
0 & -s_{3} & s_{1} s_{2} & 1+s_{1}^{2}
\end{array}\right] .
$$

In the following proposition we establish two upper bounds for the squares of singular values of the Schwarz matrix $S$.

Matrix norms will be needed in what follows. For $A=\left[a_{i j}\right] \in \mathscr{M}_{s, s}(\mathbb{C})$ we define the well-known matrix norms $\|A\|_{\infty}:=\max _{i=1,2, \ldots, s} \sum_{j=1}^{s}\left|a_{i j}\right|$ and $\|A\|_{1}:=$ $\max _{j=1,2, \ldots, s} \sum_{i=1}^{s}\left|a_{i j}\right|$. Since the matrices $R$ and $P$ are symmetric, by taking into account the form of $Q$, we conclude that the $\|\cdot\|_{1}$ and $\|\cdot\|_{\infty}$ norms of these matrices coincide; therefore, we just write $\|\cdot\|$.

Proposition 1. The singular values, $v_{j}(j=1, \ldots, m)$, of the Schwarz matrix $S$ satisfy the inequalities

$$
v_{j}^{2} \leq \frac{\|P\|+\|R\|+\sqrt{(\|P\|+\|R\|)^{2}-4\left(\|P\|\|R\|-\|Q\|^{2}\right)}}{2}
$$

and

$$
v_{j}^{2} \leq\|Q\|+\max \{\|P\|,\|R\|\} .
$$

Proof. Consider the matrix $S^{T} S=\left[\begin{array}{cc}P & Q \\ Q^{T} & R\end{array}\right]$ from Lemma 1, partitioned into the four block matrices $P, Q, Q^{T}$, and $R$. Taking the matrix norm $\|\cdot\|$ of each block of $S^{T} S$, we obtain the matricial norm of $S^{T} S$, denoted by $M\left(S^{T} S\right)$. More precisely,

$$
M\left(S^{T} S\right)=\left[\begin{array}{cc}
\|P\| & \|Q\| \\
\left\|Q^{T}\right\| & \|R\|
\end{array}\right]
$$

where

$$
\begin{gathered}
\|P\|=\max _{k=5, \ldots, m-2}\left\{\left|s_{m}^{2}\right|+\left|s_{m}\right|,\left|1+s_{m-1}^{2}\right|+\left|s_{m-1}\right|,\left|s_{k+2}\right|+\left|1+s_{k}^{2}\right|+\left|s_{k}\right|,\right. \\
\|Q\|=\max \left\{\left|s_{3}\right|,\left|s_{4}\right|\right\},\left\|Q^{T}\right\|=\|Q\|, \text { and } \\
\|R\|=\max \left\{\left|1+s_{2}^{2}\right|+\left|s_{1}\right|,\left|s_{2}\right|,\left|1+s_{1}^{2}\right|+\left|s_{1} s_{2}\right|\right\}=1+\left|s_{1} s_{2}\right|+\max \left\{s_{1}^{2}, s_{2}^{2}\right\} .
\end{gathered}
$$


Bearing in mind the relations $[\mathbf{6 , 7 , 1 1}]$

$$
v_{j}^{2} \leq \varrho\left(S^{T} S\right) \leq \varrho\left[M\left(S^{T} S\right)\right] \leq\left\|M\left(S^{T} S\right)\right\| \quad(j=1, \ldots, m)
$$

concerning the spectral radius $\varrho$, and the matricial (and matrix) norms, we must compute $\varrho\left[M\left(S^{T} S\right)\right]$ and $\left\|M\left(S^{T} S\right)\right\|$. Hence, from $\operatorname{det}\left[M\left(S^{T} S\right)-\lambda I\right]=0$, we obtain

$$
\varrho\left[M\left(S^{T} S\right)\right]=\frac{\|P\|+\|R\|+\sqrt{(\|P\|+\|R\|)^{2}-4\left(\|P\|\|R\|-\|Q\|^{2}\right)}}{2},
$$

and, applying relation (3), we get inequality (1). On the other hand, since

$$
\left\|M\left(S^{T} S\right)\right\|=\|Q\|+\max \{\|P\|,\|R\|\},
$$

applying relation (3) once again, we obtain inequality (2).

Example 1. Consider the Schwarz matrix

$$
S=\left[\begin{array}{cccc}
0 & 1 & 0 & 0 \\
1 & 0 & 1 & 0 \\
0 & 27 & 0 & 1 \\
0 & 0 & -16 & -1
\end{array}\right]
$$

Since

$$
S^{T} S=\left[\begin{array}{cccc}
1 & 0 & 1 & 0 \\
0 & 730 & 0 & 27 \\
1 & 0 & 257 & 16 \\
0 & 27 & 16 & 2
\end{array}\right]
$$

we have $P=\left[\begin{array}{cc}1 & 0 \\ 0 & 730\end{array}\right], Q=Q^{T}=\left[\begin{array}{ll}1 & 0 \\ 0 & 27\end{array}\right]$, and $R=\left[\begin{array}{cc}257 & 16 \\ 16 & 2\end{array}\right]$, with the $\infty$-norm equal to 730,27 , and 273 , respectively. In this way, applying inequality (1), for $\gamma=1, \ldots, 4$, we obtain

$$
v_{\gamma}^{2} \leq \frac{730+273+\sqrt{(730+273)^{2}-4\left(730 \times 273-27^{2}\right)}}{2}
$$

or, alternatively, $v_{\gamma}^{2} \lesssim 731.58966$, and so $v_{\gamma} \lesssim 27.047914$. Applying inequality (2), we obtain $v_{\gamma}^{2} \lesssim 27+\max \{730,273\}$ and, therefore, $v_{\gamma} \lesssim 27.514$.

Note that the singular values of $S$ calculated directly are 27.037, 16.0623, 1, and 0.0368428 .

\section{Final Remarks}

As has been said above, there are reasons justifying our interest in establishing upper bounds for the singular values of the Schwarz matrix, namely, these topics have applications in control theory. Note that inequalities (1) and (2) presented in Proposition 1 are of the Parodi type [10] and of the Carmichael and Mason type [4], respectively. 
Inequality (1) gives a bound more accurate than inequality (2), but the values involved therein are more difficult to compute.

\section{REFERENCES}

[1] Barnett, S.: Polynomials and Linear Control Systems, Marcel Dekker, Inc., New York, 1983.

[2] Berry, M. W., Drmač, Z. and Jessup, E. R.: Matrices, vector spaces, and information retrieval, SIAM Rev., 41 (1998), No. 2, 335-362.

[3] Callaerts, D., De Moor, B., Vandewalle, J., Sansen, W., Vantrappen, G. and Janssens, J.: Comparison of SVD methods to extraction of the foetal electrocardiogram from cutaneous electrode signals, Medical and Biological Engineering and Computing, 28 (1990), 217-224 .

[4] Carmichael, R. D. and Mason, T. E.: Note on the roots of algebraic equations, Bull. Amer. Math. Soc., 21 (1914), 14-22.

[5] Datta, B. N.: Numerical Linear Algebra, Cole Publishing Company, New York, 1995.

[6] Deutsch, E.: Matricial norms, Numer. Math., 16 (1970), 73-84.

[7] Fiedler, M. and Pták, V.: Generalized norms of matrices and the location of the spectrum, Czechoslovak Math. J., 12 (1962), 558-571.

[8] Genin, Y.: Hurwitz sequences of polynomials, Philips Research Reports, 30 (1975), 89-102.

[9] Lancaster, P. and Tismenetsky, M.: The Theory of Matrices, 2nd ed., Academic Press, Orlando, 1985.

[10] Parodi, M.: La Localisation des Valeurs Caractéristiques des Matrices et ses Applications, Gauthier-Villars, Paris, 1959.

[11] Robert, F. : Étude et Utilisation de Normes Vectorielles en Analyse Numérique Linéaire, Thèse, Grenoble, 1968.

[12] Schwarz, H. R.: Ein Verfahren zur Stabilitätsfrage bei Matrizen-Eigenwertproblemen, Z. Agnew. Math. Phys. 7 (1956), 473-500.

\section{Author's Address}

\section{Cecília Costa:}

Universidade de Trás-os-Montes e Alto Douro, Departamento de Matemática, 5000-911 Vila Real, Portugal

E-mail address: mcosta@utad.pt 